\title{
Density Functional Theory study of superoxide ions as impurities in alkali halides
}

Tygesen, Alexander Sougaard; Mathiesen, Nicolai Rask; Chang, Jin Hyun; García Lastra, Juan Maria

Published in:

Physical Chemistry Chemical Physics

Link to article, DOI:

$10.1039 / \mathrm{d} 0 \mathrm{cp} 00719 \mathrm{f}$

Publication date:

2020

Document Version

Peer reviewed version

Link back to DTU Orbit

Citation (APA):

Tygesen, A. S., Mathiesen, N. R., Chang, J. H., \& García Lastra, J. M. (2020). Density Functional Theory study of superoxide ions as impurities in alkali halides. Physical Chemistry Chemical Physics, 22, 13378-13389. https://doi.org/10.1039/d0cp00719f

\section{General rights}

Copyright and moral rights for the publications made accessible in the public portal are retained by the authors and/or other copyright owners and it is a condition of accessing publications that users recognise and abide by the legal requirements associated with these rights.

- Users may download and print one copy of any publication from the public portal for the purpose of private study or research.

- You may not further distribute the material or use it for any profit-making activity or commercial gain

- You may freely distribute the URL identifying the publication in the public portal 


\title{
PCCP
}

\section{ARTICLE TYPE}

Cite this: DOI: $00.0000 / x x x x x x x x x x$

\section{Density Functional Theory study of superoxide ions as impurities in} alkali halides

\author{
Alexander S. Tygesen, ${ }^{a}$ Nicolai R. Mathiesen, ${ }^{a}$ Jin Hyun Chang, ${ }^{a}$ and Juan María García- \\ Lastra*a
}

Received Date

Accepted Date

DOI: $00.0000 / x x x x x x x x x x$
The orientation of diatomic molecular impurities in crystals is a classic problem in physics, whose analysis started in the early 1930s with Pauling's pioneering studies and has extended to the present day. In the present work, we investigate the orientation of a superoxide ion $\left(\mathrm{O}_{2}{ }^{-}\right)$, which is known to be oriented in the $\langle 110\rangle$ direction when replacing a halide ion in alkali halide rock salt lattices. The unpaired electron of the superoxide, whose ground state is degenerate $\left({ }^{2} \Pi_{g}\right)$, is oriented in the $\langle 001\rangle$ direction for sodium halides while it is oriented in the $\langle 1 \overline{1} 0\rangle$ direction for potassium and rubidium halides. We performed Density Functional Theory (DFT) calculations to describe the full adiabatic potential energy surface (APES) of this complex system for the first time with ab initio methods. We are focused on four alkali halide lattices, namely $\mathrm{NaCl}, \mathrm{NaBr}, \mathrm{KCl}$, and $\mathrm{KBr}$. We show that DFT, at the Generalized Gradient Approximation (GGA) and meta-GGA levels, is able to reproduce all the experimental features for potassium halides. However, for sodium halides, although the DFT predicts the correct unpaired electron orientation, the forecasted APES energy minimum for the molecular orientation is found to be close to the $\left\langle 11^{3 / 4}\right\rangle$ orientation, in contrast to the experimental $\langle 110\rangle$ orientation. The difference in energy between the $\left\langle 11^{3 / 4}\right\rangle$ and $\langle 110\rangle$ orientation is less than $10 \mathrm{meV}$, which points out the subtleness of the considered problem. In addition to assessing the DFT accuracy and limitations to treat these systems, we also paid special attention to analyze the geometry distortions of the host lattice for the high symmetry orientations of the superoxide ion, i.e., $\langle 100\rangle,\langle 110\rangle$ and $\langle 111\rangle$. In the case of the $\langle 110\rangle$ molecular orientation, we find a strong dependence on the distance between the alkali ions in the $\langle 001\rangle$ direction and the superoxide ion upon the unpaired electron orientation. This fact explains why the orientation of the unpaired electron is different in sodium vs. potassium halides. In the case of the $\langle 100\rangle$ and $\langle 111\rangle$ molecular orientations, we analyze the Jahn-Teller vibronic coupling to find an unusually large vibronic centrifugal term in the latter.

\section{Introduction}

There has been a blooming interest on the electronic and structural properties of alkali superoxides in the past few years because of their appearance as discharge products in alkali-air batteries. ${ }^{1-3} \mathrm{KO}_{2}$ and $\mathrm{NaO}_{2}$ are the main discharge products in potassium-air $^{2}$ and sodium-air batteries ${ }^{14}$, respectively. In addition, $\mathrm{LiO}_{2}$ has been claimed to be the discharge product in Liair batteries under special circumstances, i.e., in the presence of iridium-decorated graphite. $\underline{5}$

The structure and phase transitions of alkali-superoxides were exhaustively studied by Känzig et al. in the 1970s. [6 At sufficiently high temperature stable superoxides exhibit a rock salt structure

\footnotetext{
${ }^{a}$ Department of Energy Conversion and Storage, Technical University of Denmark, 2800 Kgs. Lyngby

*E-mail: jmgla@dtu.dk, Tel: +4545258213
}

(e.g., $\mathrm{NaO}_{2}, \mathrm{KO}_{2}, \mathrm{RbO}_{2}$ and $\mathrm{CsO}_{2}$ above $293 \mathrm{~K}, 423 \mathrm{~K}, 430 \mathrm{~K}$ and $473 \mathrm{~K}$, respectively), where the superoxide $\left(\mathrm{O}_{2}{ }^{-}\right)$units are freely rotating around their center of mass. 6 It is worthwhile to note that $\mathrm{LiO}_{2}$ is not stable and decomposes to $\mathrm{Li}_{2} \mathrm{O}_{2}$ above $238 \mathrm{~K} .7$ At lower temperatures, $\mathrm{KO}_{2}, \mathrm{RbO}_{2}$ and $\mathrm{CsO}_{2}$ adopt the tetragonal structure (space group 216) with the $\mathrm{O}_{2}{ }^{-}$units rotating around the crystallographic $c$-axis. By contrast, $\mathrm{NaO}_{2}$ displays a cubic pyrite structure (space group 205) and the rotation of superoxides is limited to be only around the $\langle 111\rangle$ direction at lower temperatures. ${ }^{6}$ The origin of this different behavior of $\mathrm{NaO}_{2}$ compared with the rest of the alkali superoxides is not yet completely understood. -15 A sensible starting point for elucidating this puzzling situation is to disentangle the local behavior of a single superoxide ion (i.e., how a superoxide ion interacts with its nearest neighbor ions in the lattice) from the long-range effects (i.e., the cooperative interactions among all the superoxide ions in the lat- 
tice).

Känzig et al. 16 17 carried out electron paramagnetic resonance (EPR) studies that are instructive for understanding the local behavior of a single superoxide ion; they introduced superoxide ions as impurities in alkali halides where the $\mathrm{O}_{2}{ }^{-}$ion replaces one halide anion. The free $\mathrm{O}_{2}{ }^{-}$ion is in a degenerate electronic ${ }^{2} \Pi_{g}$ ground state, i.e., with a $\left(2 \mathrm{p} \sigma_{g}\right)^{2}\left(2 \mathrm{p} \pi_{u}\right)^{4}\left(2 \mathrm{p} \pi_{g}^{*}\right)^{3}$ configuration. The degeneracy in the $\pi_{g}^{*}$ orbitals is lifted when the $\mathrm{O}_{2}{ }^{-}$ions are embedded in a crystal lattice. Känzig's EPR experiments revealed that the superoxide ions are always oriented along the $\langle 110\rangle$ axis, irrespective of the type of the host lattice. ${ }^{16}$ Moreover, the unpaired electron is oriented along the $\langle 1 \overline{1} 0\rangle$ direction in potassium and rubidium halides while they are oriented along the $\langle 001\rangle$ direction in sodium halides (see Fig. 1). 16

(a)

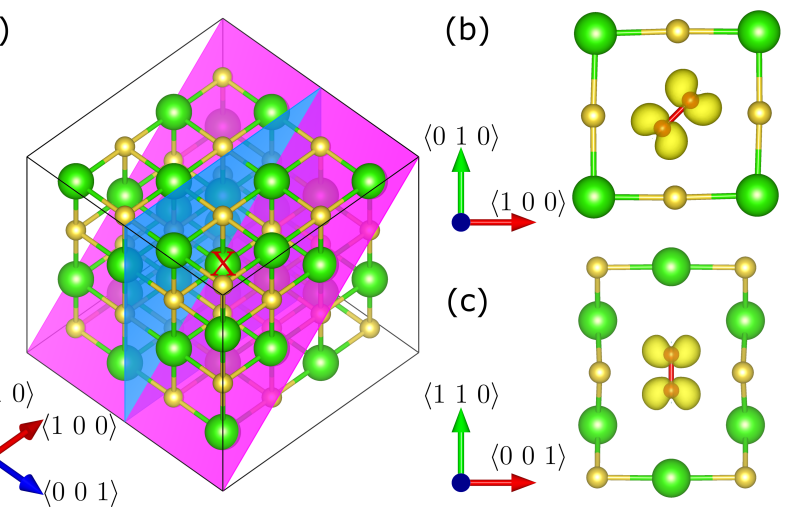

Fig. 1 (a) $2 \times 2 \times 2$ conventional supercell of a rock salt alkali halide lattice. Alkali ions are shown in yellow and halide ions are show in green. The halide ion that is substituted by a superoxide ion is marked with a red cross. The [001] and the [110] planes are shown in blue and pink, respectively. (b) Superoxide ion oriented in the $\langle 110\rangle$ direction with the unpaired electron pointing towards the $\langle 1 \overline{1} 0\rangle$ direction (the case of potassium and rubidium halides). (c) Superoxide ion oriented in the $\langle 110\rangle$ direction with the unpaired electron pointing towards in the $\langle 001\rangle$ direction (the case of sodium halides).

The simplest approach to calculate the orientation of the superoxide ion (and its unpaired electron) in rock salt structures is the general Devonshire's model ${ }^{18}$ for the rotation of molecular dimers hindered by an octahedral electric field. Devonshire's model considers the perturbation created by the electrostatic field up to the fourth-order spherical harmonics, and it can be used to predict whether the superoxide is oriented in the $\langle 111\rangle$ or $\langle 100\rangle$ orientation. Beyeler refined Devonshire's model by including sixth-order spherical harmonics in the crystal field expansion, 19 making it possible to determine if the $\langle 110\rangle$ orientation is the most preferred one. It should be mentioned that both Devonshire's and Beyeler's models neglect the possibility of having an electronic degenerate state as is the case for $\mathrm{O}_{2}{ }^{-}$. The electronic degeneracy was first considered in the model developed by Estreicher and Estle, 20 which predicts both molecular and unpaired electron orientations. The first model that goes beyond crystal field perturbation theory was proposed by Mahanti et al.21 On top of the crystal field, Mahanti's model includes the lattice distortion expressed in terms of vibrational modes and the coupling of the lattice distortion with the rotation of the molecular dimer.

All of the aforementioned models were semi-empirical, i.e., rely on parameters that can be either estimated from experimental data or taken based on a set of assumptions. The first analysis based on $a b$ initio calculations was carried out by Ewig and Tellinghuisen; they performed multiconfiguration self-consistentfield (MCSCF) calculations of the superoxide ion in vacuo and embedded in the Madelung potential of the KCl lattice. ${ }^{22}$ Their calculations showed that the superoxide oriented along the $\langle 110\rangle$ direction was energetically more preferred compared to the $\langle 100\rangle$ and $\langle 111\rangle$ directions, which is in agreement with the experiments. However, Ewig and Tellinghuisen obtained that the unpaired electron is more stable in the $\langle 001\rangle$ direction than in the $\langle 1 \overline{1} 0\rangle$ direction, which was in contrast with the experiment. ${ }^{22}$ More recently, Di Valentin and Pacchioni carried out a computational study of the EPR properties of the $\mathrm{O}_{2}{ }^{-}$ion in $\mathrm{KCl} .{ }^{23}$ They used a cluster model (explicitly including the first shells of 63 neighboring superoxide ions and surrounding that cluster with a set of point charges to represent the Madelung potential form the rest of the lattice) and Density Functional Theory (DFT) calculations at the generalized gradient approximation (GGA) and hybrid levels. The main leap forward in Di Valentin and Pacchioni's work with respect to previous studies was that the structural relaxation of the $\mathrm{O}_{2}{ }^{-}$ion surrounding first and second neighbor ions was allowed. In their study they only considered the $\langle 110\rangle$ and the $\langle 100\rangle$ molecular orientations, reporting the former to be more stable than the latter, in accordance with experiments. 23

The motivation of the present work is to shed light on why the superoxide ions behave differently as impurities in sodium halides relative to the potassium and rubidium halides. Clarifying this point may be the first step to understand why $\mathrm{NaO}_{2}$ is the only alkali superoxide that shows a cubic structure at room temperature. Using DFT calculations, our study examines the stability of the superoxide ion in various orientations, not only the high symmetry ones as was been done previously in the literature. The impact of the orientation of the half-occupied $2 \mathrm{p} \pi_{g}^{*}$ orbital on the energetics is also explicitly taken into account. This exhaustive mapping of the adiabatic potential energy surface allows the analysis of the reorientation process of the superoxide ion, i.e., the hopping of the superoxide ions from the $\langle 110\rangle$ orientation to another symmetry equivalent orientation. The latter can be compared with the available experimental results for the $\mathrm{KCl}: \mathrm{O}_{2}{ }^{-}$system, where it has been measured a faster reorientation through a $60^{\circ}$ path (e.g., from $\langle 110\rangle$ to $\langle 101\rangle$ orientation) than through a $90^{\circ}$ path (e.g., from $\langle 110\rangle$ to $\langle 1 \overline{1} 0\rangle$ orientation). 24.26

The contribution of the Jahn-Teller (JT) effect on the energy landscape and to the distortion of the lattice is another relevant issue that we analyze for the first time in this work. In the $\langle 100\rangle$ and $\langle 111\rangle$ orientations, the crystal field from the rest of the lattice is not enough to lift the electronic degeneracy, and it leads to a further distortion of the lattice that lowers the energy. It is found that a 4-fold main axis leads to an $E \otimes\left(b_{1}+b_{2}\right)$ JT coupling when the superoxide is in the $\langle 100\rangle$ orientation while a 3 -fold main axis leads to an $E \otimes(e) \mathrm{JT}$ coupling when the superoxide is

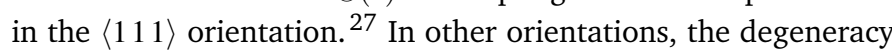
is already lifted, but the energies of the two $\mathrm{p} \pi_{g}^{*}$ orbitals are ex- 
pected to be close, and thus, a relatively strong pseudo-JT (pJT) coupling is also likely to take place.

It should be kept in mind that the spirit of the present work is to explore what can be learned from the behavior of the superoxide as an impurity to understand the phase transitions of pure alkali superoxides. For other dimer molecules such as cyanide $\left(\mathrm{CN}^{-}\right)$, there exists experimental data in which the concentration of the impurity is increased gradually from the impurity limit to the pure alkali cyanide, making it possible to estimate the local and the cooperative contributions to the phase transitions. ${ }^{1128}$ Unfortunately, that is not the case for the superoxide ion although Känzig et al. were able to study pairs of two superoxide ions placed as impurities in neighboring anionic sites. ${ }^{29]} \mathrm{A}$ natural continuation of the present work would be to perform a DFT study of superoxide pair interaction and compare it to Känzig's experimental results. However, a more fundamental question needs to be answered first: Is DFT able to reproduce the experimental results for the single impurity limit?

This paper is structured in four sections. In the Theory and Methods section, a summary of the Jahn-Teller vibronic coupling for superoxide ions oriented in $\langle 111\rangle$ and the $\langle 100\rangle$ directions is presented. Furthermore, it covers the computational methods employed in this work. The Results and Discussion section presents the results and and corresponding discussions. The paper is summarized in the Conclusions section, and some final remarks are given.

\section{Theory and Methods}

\subsection{Jahn-Teller coupling for a superoxide in a rock salt struc- ture}

The superoxide ion is a linear molecule, which in vacuo lies in the $\mathrm{D}_{\infty h}$ point symmetry group and is in a ${ }^{2} \Pi_{g}$ degenerate ground state. Its symmetry gets reduced when it is placed in an alkali halide to substitute a halide ion. The reduced point symmetry group depends on the orientation of the molecule, being $\mathrm{D}_{4 h}, \mathrm{D}_{3 d}$ and $\mathrm{D}_{2 h}$ for the high symmetry $\langle 100\rangle,\langle 111\rangle$ and $\langle 110\rangle$ orientations, respectively. ${ }^{30}$ In the $\langle 110\rangle$ orientation, the original ${ }^{2} \Pi_{g}$ degeneracy is already lifted, and the ground state can be either $\mathrm{B}_{3 g}$ (e.g., sodium halides) or $\mathrm{B}_{2 g}$ (e.g., potassium and rubidium halides). The electronic degeneracy is preserved when the superoxide ion is oriented along the $\langle 100\rangle$ or $\langle 111\rangle$ orientations. Therefore, according to the JT theorem, a further reduction of the host lattice symmetry that lowers the energy of the system must occur.

\subsection{1 $E \otimes\left(b_{1}+b_{2}\right)$ coupling in the $\langle 100\rangle$ orientation}

The superoxide ion in the $\langle 100\rangle$ orientation is in an electronic ground state within the $\mathrm{D}_{4 h}$ point symmetry group. In this situation, the electronic state couples to the $\mathrm{b}_{1 g}$ and $\mathrm{b}_{2 g}$ vibrational modes to lower its energy. The $b_{1 g}$ mode corresponds to a distortion

$$
Q_{1}= \pm \sqrt{3} a\left(\vec{Y}_{1}-\vec{Y}_{2}-\vec{Z}_{3}+\vec{Z}_{4}\right),
$$

where $\vec{Y}$ and $\vec{Z}$ correspond to the $\langle 010\rangle$ and $\langle 001\rangle$ directions, and the subscripts correspond to the labels of the alkali ions in Fig. 2. and $a$ is the magnitude of the displacement. The $\mathrm{b}_{2 g}$ mode corresponds to a distortion

$$
Q_{2}= \pm \sqrt{3} a\left(\vec{Y}_{4}-\vec{Y}_{3}+\vec{Z}_{2}-\vec{Z}_{1}\right) .
$$

In the $E \otimes\left(b_{1}+b_{2}\right) J \mathrm{~T}$ coupling, the minimum energy is always found either along the $Q_{1}$ distortion or the $Q_{2}$ distortion, but never in a linear combination of both. 31 In all the cases studied in the present work, the minimum energy is always found along the $Q_{1}$ distortion. The electronic ground state of the system for both $Q_{1}$ and $Q_{2}$ distortions is $\mathrm{B}_{2 g}$ within the $\mathrm{D}_{2 h}$ point symmetry group.
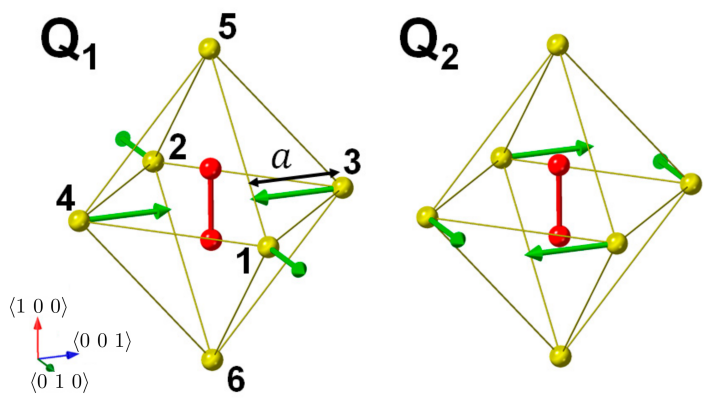

Fig. $2 E \otimes\left(b_{1}+b_{2}\right)$ coupling in the $\langle 100\rangle$ orientation. Oxygen and alkali ions are in red and yellow, respectively. Left: $Q_{1}$ mode, corresponding to Eq. (1). Right: $Q_{2}$ mode, corresponding to Eq. (2).

\subsection{2 $E \otimes(e)$ coupling in the $\langle 111\rangle$ orientation}

The superoxide ion in the $\langle 111\rangle$ orientation is in an electronic ground state within the $\mathrm{D}_{3 d}$ point symmetry group. In this situation the electronic state is coupled to two $\mathrm{e}_{g}$ vibrational modes, $Q_{\theta}$ and $Q_{\varepsilon}$, to lower its energy. $Q_{\theta}$ corresponds to a distortion

$$
\begin{aligned}
Q_{\theta}=a( & -\sqrt{3} \vec{Y}_{1}^{\prime}+\frac{3}{2} \vec{X}_{3}^{\prime}+\frac{\sqrt{3}}{2} \vec{Y}_{3}^{\prime}-\frac{3}{2} \vec{X}_{5}^{\prime}+\frac{\sqrt{3}}{2} \vec{Y}_{5}^{\prime} \\
& \left.+\sqrt{3} \vec{Y}_{2}^{\prime}-\frac{3}{2} \vec{X}_{4}^{\prime}-\frac{\sqrt{3}}{2} \vec{Y}_{4}^{\prime}+\frac{3}{2} \vec{X}_{6}^{\prime}-\frac{\sqrt{3}}{2} \vec{Y}_{6}^{\prime}\right),
\end{aligned}
$$

where $\vec{X}^{\prime}$ and $\vec{Y}^{\prime}$ correspond to the $\langle 110\rangle$ and the $\langle 1 / 21 / 2 \overline{1}\rangle$ directions, respectively. The subscripts correspond to the label of the alkali ions in Fig. 3. The $Q_{\varepsilon}$ distortion corresponds to a distortion

$$
\begin{aligned}
Q_{\varepsilon}=a( & -\sqrt{3} \vec{X}_{1}^{\prime}+\frac{\sqrt{3}}{2} \vec{X}_{3}^{\prime}-\frac{3}{2} \vec{Y}_{3}^{\prime}+\frac{\sqrt{3}}{2} \vec{X}_{5}^{\prime}+\frac{3}{2} \vec{Y}_{5}^{\prime} \\
& \left.+\sqrt{3} \vec{X}_{2}^{\prime}-\frac{\sqrt{3}}{2} \vec{X}_{4}^{\prime}+\frac{3}{2} \vec{Y}_{4}^{\prime}-\frac{\sqrt{3}}{2} \vec{X}_{6}^{\prime}-\frac{3}{2} \vec{Y}_{6}^{\prime}\right) .
\end{aligned}
$$

In this type of coupling, three equivalent energy minima separated by three saddle points are found in the so-called wrapped Mexican hat adiabatic potential energy surface (APES). ${ }^{31}$ In these minima, the point symmetry group is reduced from $\mathrm{D}_{3 d}$ to $\mathrm{C}_{2 h}$. The energy minima correspond to distort the equilateral isosceles $\widehat{135}$ and 246 in Fig. 3 , which become either elongated isosceles triangles (configurations $Q_{\theta},-\frac{1}{2} Q_{\theta}+\frac{\sqrt{3}}{2} Q_{\varepsilon}$ and $-\frac{1}{2} Q_{\theta}-\frac{\sqrt{3}}{2} Q_{\varepsilon}$ ) or shortened isosceles triangles (configurations $-Q_{\theta}, \frac{1}{2} Q_{\theta}-\frac{\sqrt{3}}{2} Q_{\varepsilon}$ 


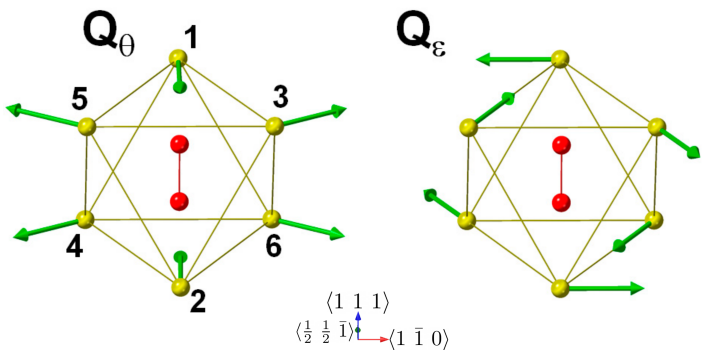

Fig. $3 E \otimes(e)$ coupling with a $\langle 111\rangle$ superoxide orientation. Oxygen and alkali ions are in red and yellow, respectively. The labeling of the alkali ions are consistent with Fig. 2 Left: $Q_{\theta}$ mode, corresponding to Eq. (3). Right: $Q_{\varepsilon}$ mode, corresponding to Eq. (4).

and $\frac{1}{2} Q_{\theta}+\frac{\sqrt{3}}{2} Q_{\varepsilon}$ ). If the triangles are elongated, the ground state of the superoxide ion in $\mathrm{B}_{g}$ while it is $\mathrm{A}_{g}$ if the triangles are shortened.

\subsection{Methods}

We studied the superoxide ion as impurity replacing an anion in four different alkali halides, namely $\mathrm{NaCl}, \mathrm{NaBr}, \mathrm{KCl}$, and $\mathrm{KBr}$. This matrix of four different compounds allows one to analyze the influence of the anion and cation choice on the superoxide orientation and energy landscape separately. All the structures considered in this work were built and analyzed using the Atomic Simulation Environment (ASE) software package. ${ }^{32}$ All the calculations were carried out using DFT as implemented in the Vienna $\mathrm{Ab}$ initio Simulation Package (VASP). 33 The Kohn-Sham one-electron valence states were expanded in a plane-wave basis using a kinetic-energy cutoff of $700 \mathrm{eV}$. The projector-augmentedwave (PAW) method was utilized to describe the atomic cores. 34 As such, 1s electrons of $\mathrm{O}$ and $\mathrm{Na}, 1 \mathrm{~s}, 2 \mathrm{~s}$ and $2 \mathrm{p}$ electrons of $\mathrm{K}$ and $\mathrm{Cl}$ and $1 \mathrm{~s}, 2 \mathrm{~s}, 2 \mathrm{p}, 3 \mathrm{~s}, 3 \mathrm{p}$ and $3 \mathrm{~d}$ electrons of $\mathrm{Br}$ were treated as core electrons. We used two different levels of theory to treat the effects of exchange and correlation: the generalized gradient approximation (GGA) using the Perdew-Burke-Ernzerhof (PBE) 35 functional and the meta-GGA approach using the Strongly Constrained and Appropriately Normed (SCAN) $\frac{36}{36}$ functional.

The first step was to determine the lattice parameter of the host lattices. These calculations were performed on the conventional rock salt unit cell using a $\Gamma$-point centered MonkhorstPack ${ }^{37} 6 \times 6 \times 6 k$-grid. All atoms were allowed to move until the Hellmann-Feynman forces were below $0.02 \mathrm{eV} / \AA$. Once that the lattice parameters for each host lattice were calculated, we proceeded with the study of the orientations of the superoxide ion and its unpaired electron.

We employed a $2 \times 2 \times 2$ conventional supercell, which comprises 65 ions (see Fig. 1), i.e., 32 alkali ions, 31 halide ions and the two oxygen ions that form the superoxide unit. Since we were calculating a doped system using a relatively large supercell, it was sufficient to consider the $\Gamma$ point only. We scanned the energies of a set of different superoxide orientations in order to obtain the APES of the system. The superoxide ions with the molecular axis pointing in the $\langle u v w\rangle$ direction were investi- gated, where $u=1, v \in\left\{0, \frac{1}{4}, \frac{1}{2}, \frac{3}{4}, 1\right\}$, and $w \in\left\{0, \frac{1}{4}, \frac{1}{2}, \frac{3}{4}, 1\right\}$ with $u \geq v \geq w$. For each orientation, we constrained the direction of the $\mathrm{O}-\mathrm{O}$ bond to be fixed. The rest of degrees of freedom of the system, including the $\mathrm{O}-\mathrm{O}$ distance, were allowed to vary during the geometry optimizations. The geometries were relaxed until all forces on atoms were lower than $0.02 \mathrm{eV} / \AA$.

An important point of the present work is the analysis of the orientation of the unpaired electron. Thus, for a given molecular orientation $\langle u v w\rangle$, it is crucial to calculate the energy difference between the unpaired electronic orientation for which the energy is minimized. We denote the orientation of the molecular orientation of the superoxide with the minimum energy as $\langle u v w\rangle_{\min }$ and the unpaired electronic orientation perpendicular to $\langle u v w\rangle_{\min }$ as $\langle u v w\rangle_{\text {per. }}$ The geometry and energy of the $\langle u v w\rangle_{\text {min }}$ configuration is obtained naturally by relaxing the geometries with the imposed $\langle u v w\rangle$ superoxide orientation. However, obtaining the geometry and energy of the $\langle u v w\rangle_{\text {per }}$ configuration is much more involved and requires the following steps:

1. Obtain the geometry for the $\langle u v w\rangle_{\min }$ configuration, $\mathbf{R}_{(u, v, w)_{\min }}$.

2. Obtain the geometry of an electronic configuration in which the unpaired electron is equally split between the two $2 \mathrm{p} \pi_{g}^{*}$ orbitals, $\mathbf{R}_{(u, v, w)_{1 / 2}}$. This can be done in VASP by setting the electronic occupations of these two orbitals to $1 / 2$ and $1 / 2$. In several studies on Jahn-Teller systems, this electronic configuration is often called "the artificial configuration". 38 Here, we denote it as a $\langle u v w\rangle_{1 / 2}$ configuration.

3. Once $\mathbf{R}_{(u, v, w)_{\min }}$ and $\mathbf{R}_{(u, v, w)_{1 / 2}}$ are determined, build a good initial guess for the geometry of the $\langle u v w\rangle_{\text {per }}$ configuration, $\mathbf{R}_{(u, v, w) \text { per }}^{\text {init }}$, by calculating the atomic displacements from $\mathbf{R}_{(u, v, w)_{1 / 2}}$ to $\mathbf{R}_{(u, v, w)_{\min }}$ configuration and then applying the same displacement to the $\mathbf{R}_{(u, v, w)_{1 / 2}}$ configuration with an opposite sign, i.e.,

$$
\begin{aligned}
\mathbf{R}_{(u, v, w)_{\text {per }}}^{\text {init }} & =\mathbf{R}_{(u, v, w)_{1 / 2}}-\left(\mathbf{R}_{(u, v, w)_{\min }}-\mathbf{R}_{(u, v, w)_{1 / 2}}\right) \\
& =2 \mathbf{R}_{(u, v, w)_{1 / 2}}-\mathbf{R}_{(u, v, w)_{\min }}
\end{aligned}
$$

4. Perform a geometry optimization starting from $\mathbf{R}_{(u, v, w)_{\text {per }}}^{\text {init }}$ to obtain $\mathbf{R}_{(u, v, w)}$ per

Apart from obtaining $\mathbf{R}_{(u, v, w)_{\text {per }}}$, the calculations on $\mathbf{R}_{(u, v, w)_{1 / 2}}$ allow one to assess how important the orientation of the unpaired electron is to determine the final orientation of the superoxide ion. If we were to compare our $a b$ initio results with the crystal field model, the $\langle u v w\rangle_{1 / 2}$ configuration would represent Beyeler's model while the $\langle u v w\rangle_{\min }$ would represent Estreicher and Estle's model.

\section{Results and Discussion}

Table 1 shows the calculated lattice parameters for the pure rock salt host lattices using SCAN and PBE exchange-correlation (xc) functionals. It can be observed that PBE overestimates lattice parameters while SCAN underestimates them. The mean absolute 
error (MAE) is slightly larger for PBE than for SCAN (i.e. $\sim 8 \mathrm{pm}$ for PBE and $\sim 5 \mathrm{pm}$ for SCAN). Despite the difference in the assessment of the lattice parameters, the qualitative results for the preferred orientation of the superoxide ion and the relative energies of the high symmetry orientations $(\langle 100\rangle,\langle 110\rangle$, and $\langle 111\rangle)$ are very similar. Both xc-functionals predict the minimum energy orientation to be $\langle 110\rangle$ for the potassium halides, in agreement with experiments, and $\left\langle 11^{3} / 4\right\rangle$ for the sodium halides (the minimum energy orientation of $\mathrm{NaCl}$ using $\mathrm{PBE}$ is $\left\langle 11^{1 / 2}\right\rangle$, but is only $1.3 \mathrm{meV}$ more stable than the $\left\langle 11^{3 / 4}\right\rangle$ orientation). In the case of sodium halides, both PBE and SCAN predict the experimentally observed most stable $\langle 110\rangle$ orientation to be $\sim 10 \mathrm{meV}$ above the minimum energy in the APES. Another similarity between both xc-functionals is that the $\langle 100\rangle$ orientation is the most unstable one with energies above the minimum in the APES of $\sim 250 \mathrm{meV}$ and $\sim 50 \mathrm{meV}$ for the sodium and the potassium halides, respectively. The remaining discussion is based on the results from the SCAN functional as the two functionals lead to very similar results, while SCAN has smaller MAE for the lattice parameters.

Table 1 Calculated lattice parameters, position of the minimum energy superoxide orientation in the APES and the energies of the high symmetry orientations (relative to that of the minimum) using the PBE and SCAN xc-functionals

\begin{tabular}{clcccc}
\hline Species & & $\mathrm{NaCl}$ & $\mathrm{NaBr}$ & $\mathrm{KCl}$ & $\mathrm{KBr}$ \\
\hline \multirow{2}{*}{$\mathrm{LP}(\AA)$} & $\mathrm{PBE}$ & 5.697 & 6.035 & 6.384 & 6.705 \\
& $\mathrm{SCAN}$ & 5.513 & 5.924 & 6.285 & 6.571 \\
& $\mathrm{Exp}^{b}$ & 5.64 & 5.978 & 6.294 & 6.596 \\
\hline Favored & $\mathrm{PBE}$ & $\left\langle 11^{3} / 4\right\rangle$ & $\left\langle 11^{3} / 4\right\rangle$ & $\langle 110\rangle$ & $\langle 110\rangle$ \\
Orientation & $\mathrm{SCAN}$ & $\langle 111 / 2)$ & $\left\langle 11^{3} / 4\right\rangle$ & $\langle 110\rangle$ & $\langle 110\rangle$ \\
\hline$\langle 100\rangle$ energy & $\mathrm{PBE}$ & 278 & 255 & 64 & 56 \\
(meV) & $\mathrm{SCAN}$ & 257 & 236 & 40 & 50 \\
\hline$\langle 110\rangle$ energy & $\mathrm{PBE}$ & 13 & 10 & 0.0 & 0.0 \\
(meV) & $\mathrm{SCAN}$ & 7 & 9 & 0.0 & 0.0 \\
\hline$\langle 111\rangle$ energy & PBE & 10 & 11 & 23 & 10 \\
(meV) & SCAN & 15 & 8 & 38 & 4 \\
\hline
\end{tabular}

$b$ Experimental data taken from Ref. 39

$c$ The $\left\langle 11^{3 / 4}\right\rangle$ orientation is only $1.3 \mathrm{meV}$ above the $\left\langle 11^{1 / 2} / 2\right\rangle$ orientation

APES for the four systems are illustrated in Fig. 4. which shows the orientation of the unpaired electron for each superoxide orientation in addition to the main features of the energy landscape discussed above. Our calculations predict that the superoxide molecule in the $\langle 110\rangle$ orientation has its unpaired electron oriented in the $\langle 001\rangle$ direction for the sodium halides and oriented in the $\langle 1 \overline{1} 0\rangle$ direction for the potassium halides, which agrees well with experiments. 1617 As a salient feature, the orientation of the unpaired electron in sodium halides is perpendicular to that in potassium halides for every considered superoxide orientation with the exception of $\langle 100\rangle$ and $\langle 111\rangle$ superoxide orientations where JT effect takes place.

Regarding the barriers for reorientation hopping among the symmetry equivalent $\langle 110\rangle$ superoxide orientations, two possibilities have been considered in the literature:25|26]40 A $90^{\circ}$ reorientation (e.g., from $\langle 110\rangle$ orientation to $\langle 1 \overline{1} 0\rangle$ orientation
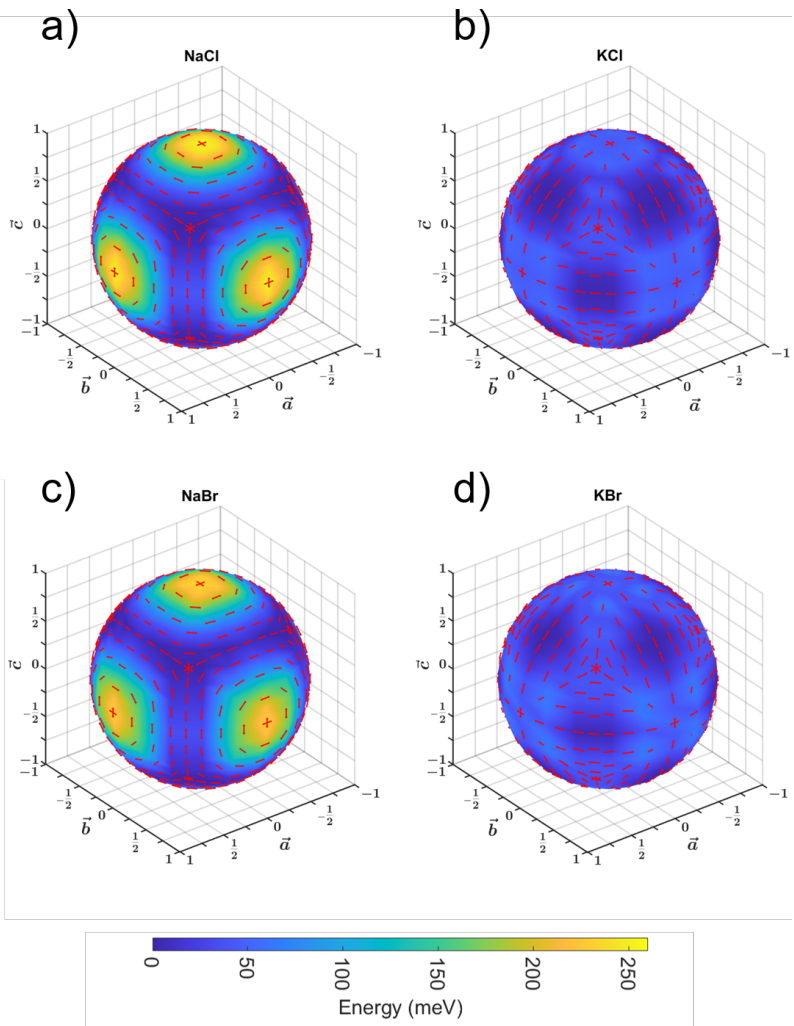

Fig. 4 APES as a function of the superoxide ion orientation for a) $\mathrm{NaCl}$, b) $\mathrm{KCl}$, c) $\mathrm{NaBr}$ and d) $\mathrm{KBr}$. The red lines indicate the orientation of the unpaired electron at each superoxide orientation.

through the $\langle 100\rangle$ orientation) and a $60^{\circ}$ reorientation (e.g., from $\langle 110\rangle$ orientation to $\langle 101\rangle$ orientation through the vicinity of the $\left\langle 1^{1 / 2} 1 / 2\right\rangle$ orientation). Here, we observe that minimum energy barriers for the potassium halides (see Figs. 4, 6(b), and 8(b)) occur for the $\left\langle 13 / 4^{3} / 4\right\rangle$ orientation, both for $\mathrm{KCl}(39.7 \mathrm{meV}$ barrier) and $\mathrm{KBr}$ (37.5 meV barrier), which is identified as the point in the vicinity of the $\left\langle 1^{1 / 2} 1^{1 / 2}\right\rangle$ orientation. The barriers for the $90^{\circ}$ reorientation through the $\langle 100\rangle$ orientation are $45.9 \mathrm{meV}$ and $49.9 \mathrm{meV}$ for $\mathrm{KCl}$ (note that for $\mathrm{KCl}$ the transition state occurs at the $\langle 11 / 40\rangle$ orientation, slightly before reaching the $\langle 100\rangle$ orientation) and $\mathrm{KBr}$, respectively. The preference of the $60^{\circ}$ reorientation over the $90^{\circ}$ reorientation for $\mathrm{KCl}$ is in agreement with the phenomenological interpretation of Känzig on his experiments for $\mathrm{KCl}$ and $\mathrm{KI} .25$ Känzig also suggested that the difference in the energy barrier between the $60^{\circ}$ and $90^{\circ}$ reorientations should increase as the halide ion size increases due to steric effects, which is also well-aligned with the results presented here.

It was briefly mentioned in the Introduction that the model by Estreicher and Estle 20 is an improvement of the model by Beyeler 19 as it accounts for the fact that the degeneracy of the ${ }^{2} \Pi_{g}$ ground state for superoxides should be lifted. The impact of the degeneracy lifting on the APES can be evaluated by comparing the energy landscape of the artificial states in which both ${ }^{2} \Pi_{g}$ orbitals are occupied by 1.5 electrons to that of the real nondegenerate ground state for every superoxide orientation (Figs. 5 to 8 . It can be seen from Figs. 5 to 8 that the minimum-energy 

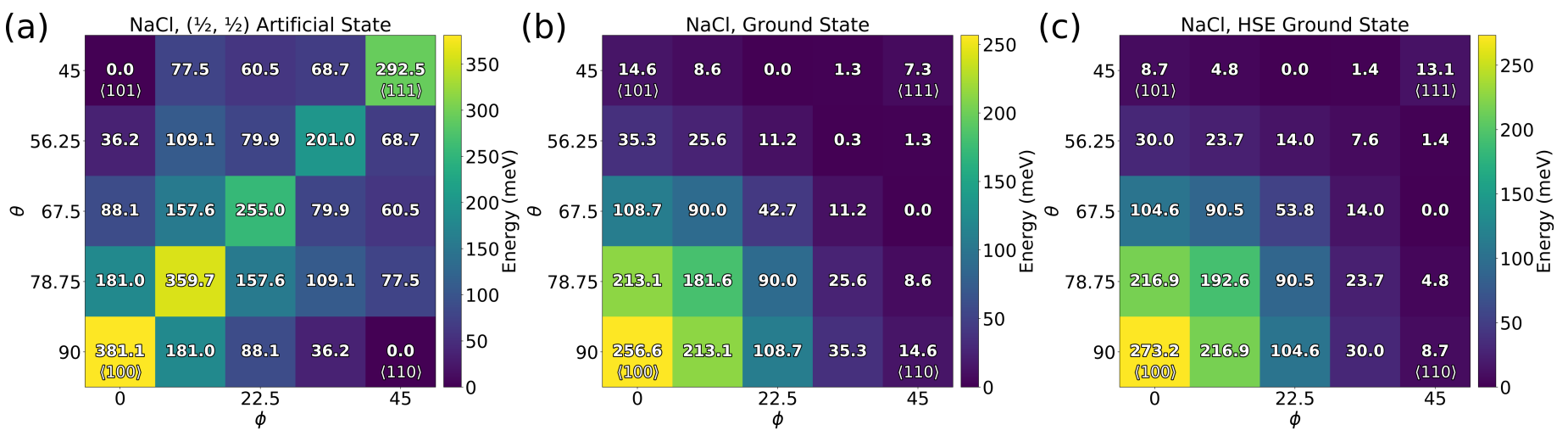

Fig. 5 Calculated APES (in polar coordinates) using SCAN xc-functional of the $\mathrm{NaCl}: \mathrm{O}_{2}{ }^{-}$system for a) the (1/2, $1 / 2$ ) artificial state and b) the real ground state. c) shows the calculated real ground state using the HSE hybrid xc-functional. All the energies are given with respect to the minimum energy point in each plot.
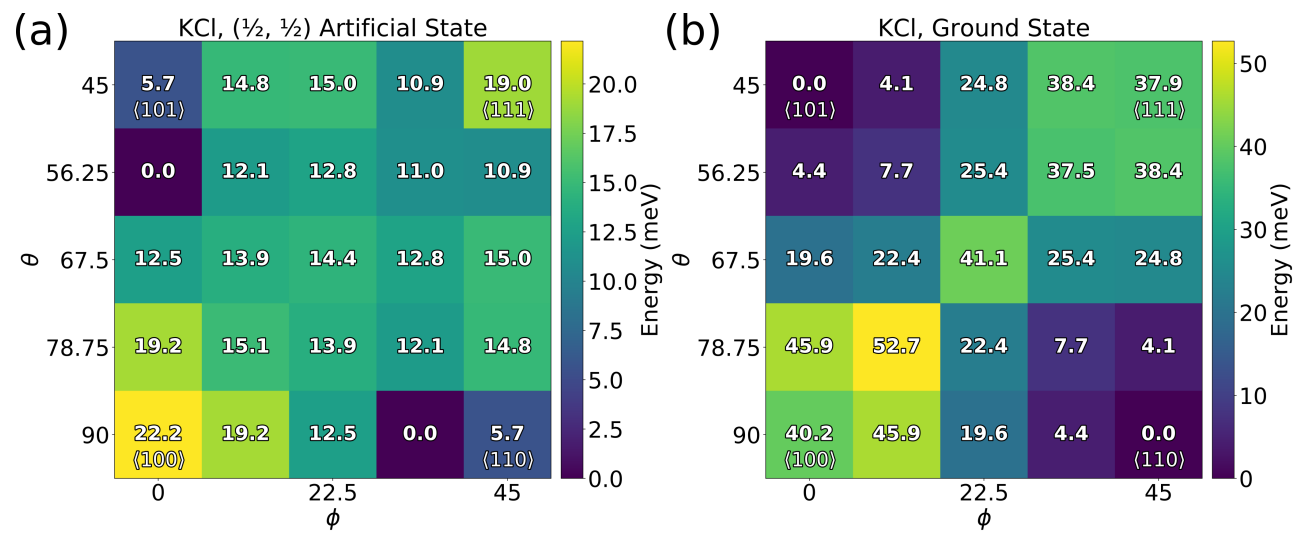

Fig. 6 Calculated APES (in polar coordinates) using SCAN xc-functional of the $\mathrm{KCl} \mathrm{O}_{2}{ }^{-}$system for a) the (1/2, $\left.1 / 2\right)$ artificial state and b) the real ground state. All the energies are given with respect to the minimum energy point in each plot.

orientations of the superoxide is different for the artificial and real non-degenerate states for all considered systems. In potassium halides, the artificial state minima are at the $\langle 13 / 40\rangle$ and $\langle 111\rangle$ orientations for $\mathrm{KCl}$ and $\mathrm{KBr}$, respectively. In sodium halides, the artificial state minima are at the $\langle 110\rangle$ and $\langle 111\rangle$ orientations for $\mathrm{NaCl}$ and $\mathrm{NaBr}$, respectively. It appears that the energy minima in the artificial state are governed by the halide nature: alkali bromides have the energy minima at the $\langle 111\rangle$ orientation while alkali chlorides have the energy minima at the $\langle 110\rangle$ orientation or close to it (the artificial state energy minima of $\mathrm{KCl}$ is only $5.7 \mathrm{meV}$ above the $\langle 110\rangle$ ). This is in contrast with the real nondegenerate state, where the orientation of the energy minima is dictated by the alkali ion. Despite this disagreement between the energy minima in the artificial and the real non-degenerate states, there are also important similarities between them: In both cases the energy maxima are at the $\langle 100\rangle$ orientation, and the magnitude of the energy span of the APES is similar in both cases when passing from the artificial to the real non-degenerate state is kept. This points out that the instability of the $\langle 100\rangle$ direction is captured without considering the orientation of the unpaired electron. However, more subtle features such as the position of the energy minima require that the unpaired electron orientation be taken into account explicitly.

The importance of the unpaired electron orientation for the su- peroxide in the $\langle 110\rangle$ orientation is apparent. For all the considered systems, the energy difference between the $\langle u v w\rangle_{\min }$ (the electronic orientation with minimum energy) and $\langle u v w\rangle_{\text {per }}$ (the electronic orientation perpendicular to $\left.\langle u v w\rangle_{\min }\right), \Delta E_{\min -\text { per }}$, is maximized in the $\langle 110\rangle$ orientation. Table 2 shows the magnitude of $\Delta E_{\text {min-per }}$ which spans from $67 \mathrm{meV}$ in $\mathrm{KBr}$ to $105 \mathrm{meV}$ in $\mathrm{NaBr}$. It can be seen that $\langle u v w\rangle_{\min }=\langle 001\rangle$ for sodium halides while $\langle u v w\rangle_{\min }=\langle 1 \overline{1} 0\rangle$ for potassium halides, in agreement with experimental results. $\frac{16] 17}{16}$ Looking at Table 2, it can be noticed that when $\langle u v w\rangle_{\min }=\langle 001\rangle$, the distance between center of mass of the superoxide ion and the four alkali ions lying in the [001] plane, $R^{\text {eq }}$, is similar to the distance to the two nearest alkali ions out of the [001] plane (i.e., those along the $\langle 001\rangle$ direction), $R^{\text {ax }}$ (see Figure 9). However, when $\langle u v w\rangle_{\min }=\langle 1 \overline{1} 0\rangle, R^{\mathrm{eq}}$ is significantly larger than $R^{\text {ax }}$ (more than $30 \mathrm{pm}$ in all the cases). This variation in the difference between $R^{\mathrm{ax}}$ and $R^{\mathrm{eq}}$ is mainly due to the changes in $R^{\mathrm{ax}}$ since $R^{\mathrm{eq}}$ is relatively close to the alkali-halide distance in the pure host lattices $(a / 2$ in Table 3$)$ for all the alkali halides and all unpaired electron orientations. The reason for this behavior is obvious: There are two electrons pointing directly towards the axial alkali ions for $\langle u v w\rangle_{\min }=\langle 1 \overline{1} 0\rangle$ while there is only one electron for $\langle u v w\rangle_{\min }=\langle 001\rangle$, which substantially decreases the electrostatic attraction in the latter vs. the former. 41 However, there are no $\mathrm{p}$ electrons directly pointing towards equa- 

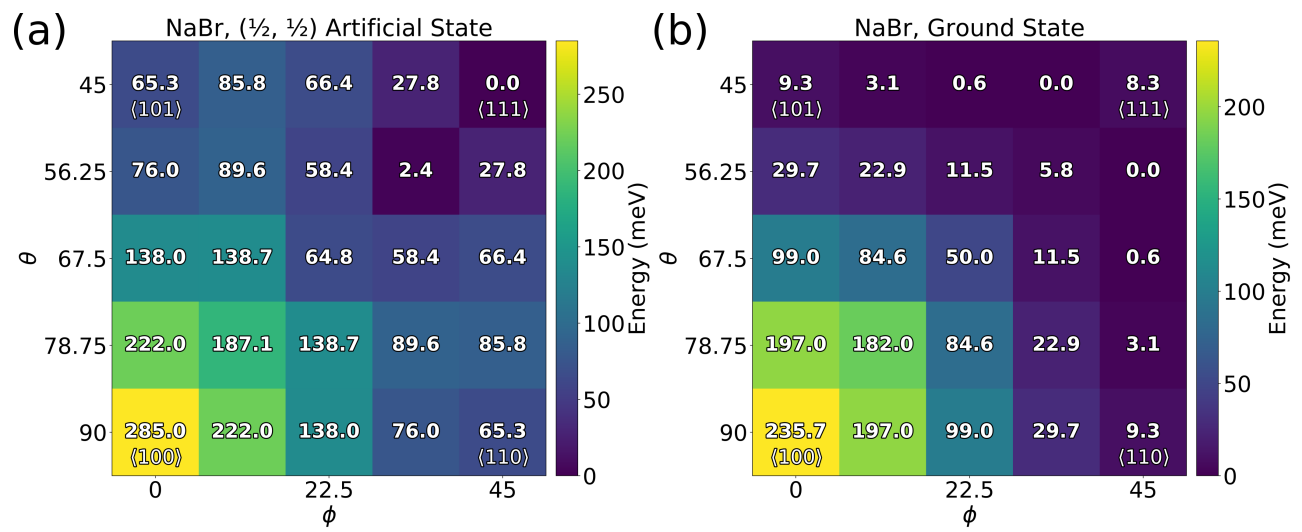

Fig. 7 Calculated APES (in polar coordinates) using SCAN xc-functional of the $\mathrm{NaBr}: \mathrm{O}_{2}^{-}$system for a) the (1/2, 1/2) artificial state and b) the real ground state. All the energies are given with respect to the minimum energy point in each plot.
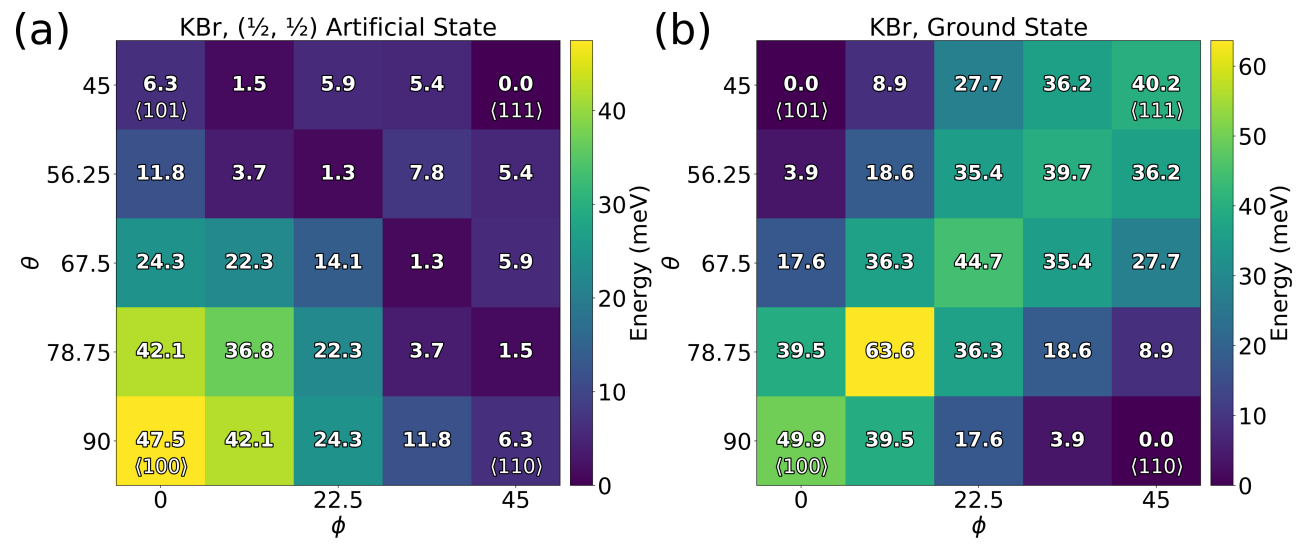

Fig. 8 Calculated APES (in polar coordinates) using SCAN xc-functional of the $\mathrm{KBr}: \mathrm{O}_{2}{ }^{-}$system for a) the $(1 / 2,1 / 2)$ artificial state and b) the real ground state. All the energies are given with respect to the minimum energy point in each plot.

torial alkali ions regardless of the orientation, $\langle u v w\rangle_{\min }$. Since $R^{\text {ax }}$ is heavily dependent on $\langle u v w\rangle_{\min }$, it seems natural to think that, following Mahanti's model, the elastic interactions between the superoxide ion and the host lattice may play an important role in determining which unpaired electron orientation is preferred. Indeed, sodium halides are $\sim 35 \%$ harder than their potassium counterparts 42 , making it less energetically favorable to have a large compression in $R^{\text {ax }}$ when $\langle u v w\rangle_{\min }=\langle 1 \overline{1} 0\rangle$. Thus, this could be eventually one of main causes for the stabilization of the $\langle u v w\rangle_{\min }=\langle 001\rangle$ in sodium halides.

Table 2 Equatorial, $R^{\text {eq }}$, and axial, $R^{\text {ax }}$, distances calculated for the superoxide oriented in the $\langle 110\rangle$ direction for the unpaired electron oriented in the $\langle 001\rangle$ and $\langle 1 \overline{1} 0\rangle$ directions. The calculated energy difference between the two unpaired electron orientations is also shown. All values are calculated using SCAN xc-functional

\begin{tabular}{lccccc}
\hline Species & $\begin{array}{c}R_{\langle 1 \overline{1} 0\rangle}^{\mathrm{eq}} \\
(\AA)\end{array}$ & $\begin{array}{c}R_{\langle 1 \overline{1} 0\rangle}^{\mathrm{ax}}(\AA) \\
\mathrm{NaCl}\end{array}$ & $\begin{array}{c}R_{\langle 001\rangle}^{\mathrm{eq}} \\
(\AA)\end{array}$ & $\begin{array}{c}R_{\langle 001\rangle}^{\mathrm{ax}} \\
(\AA)\end{array}$ & $\begin{array}{c}\Delta E_{\min -p e r} \\
(\mathrm{meV})\end{array}$ \\
$\mathrm{NaBr}$ & 2.957 & 2.500 & 2.768 & 2.661 & 98.8 \\
$\mathrm{KCl}$ & 3.177 & 2.512 & 2.870 & 2.860 & 104.7 \\
$\mathrm{KBr}$ & 3.272 & 2.841 & 3.168 & 2.994 & -86.6 \\
\hline
\end{tabular}

We have also evaluated the magnitude of the JT distortions when the superoxide ion is oriented in $\langle 100\rangle$ and $\langle 111\rangle$ directions. For the $\langle 100\rangle$ orientation where $E \otimes\left(b_{1}+b_{2}\right)$ JT coupling takes place, our calculation setup only allows one to analyze the distortions along the $Q_{1}$ mode where the energy minima are found, but not the distortions along the $Q_{2}$ mode where the saddle point between the two $Q_{1}$ minima are found. The magnitude of the $Q_{1}$ distortion can be written in the first approximation as $Q_{1}^{\min }=F^{2} / K$, where $F$ and $K$ are the vibronic coupling constant and the force constant for the $Q_{1}$ mode, respectively. ${ }^{27}$ In general, both $F^{2}$ and $K$ decrease as the lattice parameter is increased. It can be seen from from Table 4 that the magnitude of the $Q_{1}$ distortion decreases as we move from sodium halides to potassium halides and from alkali bromides to alkali chlorides, which indicates that $F^{2}$ decreases faster than $K$ with the increase in lattice parameter. Regarding the $E \otimes(e)$ coupling in the $\langle 111\rangle$ orientation, the JT distortions are significantly smaller than the $E \otimes\left(b_{1}+b_{2}\right)$ JT coupling (see Table 4) mainly because the active electrons involved in the JT coupling in the latter are more oriented towards the alkali ions. In the case of the $E \otimes(e)$ coupling, we were able to determine the energy barriers, $E_{b}$, between the three equivalent $Q_{\theta}$ energy minima (in the $\langle u v w\rangle_{\min }$ configuration by definition) since the three equivalent $Q_{\varepsilon}$ energy saddle points are found in the $\langle u v w\rangle_{\text {per }}$ configuration. We found that $E_{b}$ is very small in all the cases, i.e., $E_{b}<2 \mathrm{meV}$, which implies that 
Table 3 Average distance between the superoxide center of mass and its six nearest alkali ions, $\bar{R}$ (in $\AA$ ) for the high symmetry superoxide orientations. The difference between $\bar{R}$ and half of the lattice parameter of the pure alkali halides $(a / 2), \Delta R$, is also shown. $\Delta R_{\exp }$ is the difference in the experimental half lattice parameter between the pure alkali halide and the pure alkali superoxide in its cubic high temperature phase

\begin{tabular}{lcccccccc}
\hline & $a / 2$ & $\bar{R}_{\langle 100\rangle}$ & $\bar{R}_{\langle 110\rangle}$ & $\bar{R}_{\langle 111\rangle}$ & $\Delta R_{\langle 100\rangle}$ & $\Delta \bar{R}_{\langle 110\rangle}$ & $\Delta \bar{R}_{\langle 111\rangle}$ & $\Delta R_{\exp }$ \\
\hline $\mathrm{NaCl}$ & 2.756 & 2.700 & 2.731 & 2.736 & 0.056 & 0.025 & 0.020 & 0.075 \\
$\mathrm{NaBr}$ & 2.962 & 2.822 & 2.867 & 2.854 & 0.140 & 0.095 & 0.108 & 0.244 \\
$\mathrm{KCl}$ & 3.142 & 3.064 & 3.068 & 3.114 & 0.078 & 0.074 & 0.028 & 0.102 \\
$\mathrm{KBr}$ & 3.285 & 3.134 & 3.130 & 3.184 & 0.151 & 0.155 & 0.101 & 0.253 \\
\hline
\end{tabular}

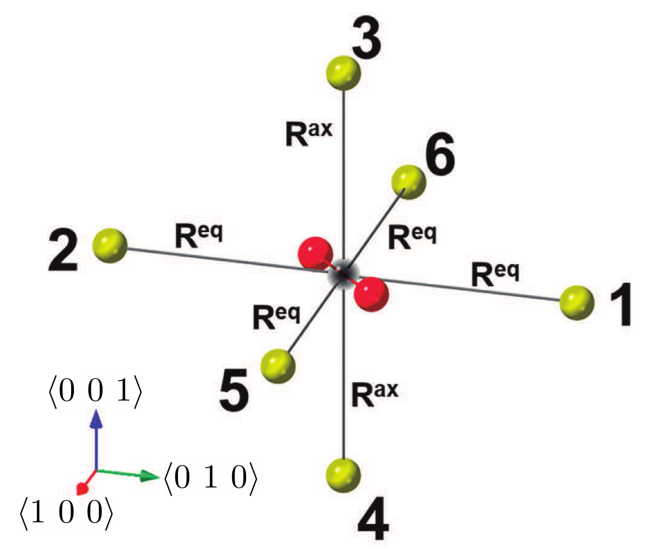

Fig. 9 Geometry of the octahedron formed by the six alkali ions surrounding the superoxide ion when it is oriented in the $\langle 110\rangle$ direction. Oxygen and alkali ions are in red and yellow, respectively. The center of mass of the superoxide ion is marked with a grey sphere. The four alkali ions in the [001] plane are at a distance $R^{\text {eq }}$ from the center of mass of the superoxide ion. The two ions in the $\langle 001\rangle$ direction are at a distance $R^{\text {ax }}$ from the center of mass of the superoxide ion.

we are in a dynamic JT case with coherent tunneling between the three minima at low temperatures.

Table 4 Calculated magnitude of the Jahn-Teller distortion in $\langle 100\rangle$ and $\langle 111\rangle$ orientations (magnitude of the displacements $a$ in Figs. 2 and 3 ) using SCAN xc-functional. The energy barrier, $E_{b}$, for hopping from each of the three equivalent energy minima in the wrapped Mexican hat of the $E \otimes(e) \mathrm{JT}$ coupling in the $\langle 111\rangle$ orientation is also shown

\begin{tabular}{lccc}
\hline Species & $\begin{array}{c}a \text { in }\langle 100\rangle \\
Q_{1}(\mathrm{pm})\end{array}$ & $\begin{array}{c}a \text { in }\langle 111\rangle \\
Q_{\theta}(\mathrm{pm})\end{array}$ & $\begin{array}{c}E_{b} \text { in }\langle 111\rangle \\
(\mathrm{meV})\end{array}$ \\
\hline $\mathrm{NaCl}$ & 11 & 8 & 0.1 \\
$\mathrm{NaBr}$ & 23 & 4 & 1.8 \\
$\mathrm{KCl}$ & 9 & 5 & 0.4 \\
$\mathrm{KBr}$ & 17 & 3 & 0.1 \\
\hline
\end{tabular}

There still remains an unanswered fundamental question: Why is the present model unable to predict the right energy minima for sodium halides? Is it because of the intrinsic limitations of DFT, or is it because some real physical effects have not been taken into account? It should be noted that the energy differences between the line that connects the $\langle 110\rangle$ and $\langle 111\rangle$ orientations are rather small, i.e., $\langle 15 \mathrm{meV}$ and $\langle 10 \mathrm{meV}$ for the $\langle 110\rangle$ and $\langle 111\rangle$ orientations, respectively. Therefore, small corrections for the effects such as spin-orbit coupling or zero-phonon energies (ZPE) could change the exact positions of the global minima in the APES.

We checked the presence of some pitfalls of DFT calculations by looking at the $\mathrm{O}-\mathrm{O}$ distance of the superoxide ions, $R_{\mathrm{O}-\mathrm{O}}$, in order to determine if there are any significant differences for different orientation and/or host lattices. For all cases, the $\mathrm{O}-\mathrm{O}$ distances are $1.35 \pm 0.01 \AA$, which is very close to the results obtained by Ewig and Tellinghuisen in their MCSCF study where they found $R_{\mathrm{O}-\mathrm{O}}=1.340 \pm 0.005 \AA$ for the four studied configurations. 22 We also considered the possibility of a change in the energy minima position due to spin-orbit interactions; we performed spin-orbit calculations with VASP along all the orientations along the $\langle 110\rangle$ to $\langle 111\rangle$ line. We found that the spin-orbit only produces a rigid shift in the energies (i.e., the relative energy variation of the total energy due to spin-orbit effects is less than $1 \mathrm{meV}$ ). The possibility of the vibrational modes of the lattice getting affected by the orientation of the superoxide impurity, similar to the rotational-vibrational coupling in Mahanti's model ${ }^{21}$, is also considered. In practice we did that by calculating the change in the ZPEs of the lattice for each superoxide orientation (only the movement of the 26 first nearest neighbors to the superoxide ion was allowed in the ZPE calculations). Although this produces slight variations in the APES (not shown), the energy minima are still not found at the $\langle 110\rangle$ orientation. Finally, we evaluated the magnitude of the so-called vibronic centrifugal term, 43 which destabilizes configurations close to a conical intersection in a given APES. A relatively large vibronic centrifugal term is found for the $\langle 111\rangle$ orientation where the JT distortion were found to be particularly small. The destabilization energy due to the vibronic centrifugal term, $E_{\text {cent }}$, is written in atomic units as

$$
E_{\text {cent }}=\frac{1}{8 M \rho^{2}},
$$

where $M$ is the reduced mass of the vibrational mode $(M=23$ Dalton in this case) and $\rho$ is the magnitude of the distortion $\left(Q_{\theta}\right.$ in our case), $\rho=\sqrt{3} a$. For the case of $\mathrm{NaCl}$ where $a=8 \mathrm{pm}$ then $E_{\text {cent }} \approx 1 \mathrm{meV}$. On the other hand, $\mathrm{NaBr}$ with $a=4 \mathrm{pm}$ we find $E_{\text {cent }} \approx 4 \mathrm{meV}$. However, even in the case where $E_{\text {cent }}$ is similar in the $\left\langle 11^{3 / 4}\right\rangle$ to that of the $\langle 111\rangle$ orientation due to its proximity, destabilization energy contribution is not enough to make the $\langle 110\rangle$ orientation the global minimum in the APES.

In view that none of the physical effects considered above can account for the fact that the energy minima in the APES are not found at the $\langle 110\rangle$ orientation for sodium halides, we repeated our calculations employing a higher level of theory, namely using the Heyd-Scuseria-Ernzerhof (HSE06) ${ }^{44}$ functional. Due to its computational cost, we only repeated the calculations for the $\mathrm{NaCl}$ system. Fig. 1)(c) shows the HSE06 results, which barely changes the energy landscape when compared to the SCAN or PBE results, confirming the robustness of the model against the 
choice of the xc-functional.

\section{Conclusions}

We have revisited a classic problem of solid-state physics, namely the orientation and rotation of diatomic molecular impurities in rock salt lattices 45 , which had become nearly forgotten nowadays. The seminal theoretical papers by Pauling 46 and Devonshire ${ }^{18}$ in the 1930 s were followed by a plethora of experimental data, mainly gathered between the 1950 s to the 1970 s. $\frac{45}{\text { The }}$ level of complexity of the models used to interpret these experimental results was increasing gradually $12 \mid 18-20$, building up to the dynamical models by Kiljunen et al.47/48 Nevertheless, no mapping of the APES of this type of system using $a b$ initio electronic structure methods had been reported. Here, we investigate the superoxide ion as an impurity in four rock salt systems using DFT. The system is quite challenging since the ground state of free superoxide ions is double degenerate. However, the choice was justified as understanding the impurity limit could lead to rationalizing the phase transitions observed in pure alkali superoxides, materials that have gained much attention in the field of alkali metal-air batteries.

One of the key points in our study is to check how much of the experimental data could be reproduced by the DFT model. For each system, there were two relevant features to consider, namely the orientation of the superoxide molecule and the orientation of its unpaired electron. We showed that DFT at the GGA and metaGGA levels can correctly reproduce all the experimental data for potassium halides, including the superoxide ion reorientation in KCl. By contrast, DFT, even at the hybrid level, is unable to reproduce the experimental orientation of the superoxide ions in sodium halides. However, we found that the DFT models predict energies only $\sim 10 \mathrm{meV}$ above the energy minima of the APES. We verified that physical effects not considered explicitly in the model such as spin-orbit coupling, the ZPE of the lattice for each specific molecular orientation and the vibronic centrifugal term (which is unusually large close to the $\langle 111\rangle$ molecular orientation), are not able to account for the $10 \mathrm{meV}$ energy discrepancy. For this limit of small energies, it is well known that DFT methods can readily fail on predicting minimum energy configurations and that more accurate approaches such as post-Hartree-Fock methods are required. 49

Despite the limitation in the DFT accuracy, we show the strong impact of the orientation of the unpaired electron on the local environment of the superoxide ion (i.e., the first shell of its alkali nearest neighbors) oriented in the $\langle 110\rangle$ direction. We conclude that the elastic coupling of the superoxide with the host lattice plays a key role in stabilizing the unpaired electron in the $\langle 001\rangle$ orientation in potassium halides vs. the preferred $\langle 1 \overline{1} 0\rangle$ orientation in sodium halides.

\section{Conflicts of interest}

There are no conflicts to declare.

\section{Acknowledgements}

The authors acknowledge support from the Villum Foundation's Young Investigator Programme $\left(4^{\text {th }}\right.$ round, project: In silico design of efficient materials for next generation batteries. Grant number: 10096).

\section{Notes and references}

1 K. Song, D. A. Agyeman, M. Park, J. Yang and Y.-M. Kang, Adv. Mater., 2017, 29, 1606572.

2 N. Xiao, X. Ren, W. D. McCulloch, G. Gourdin and Y. Wu, Acc. Chem. Res., 2018, 51, 2335-2343.

3 N. R. Mathiesen, S. Yang, J. M. García-Lastra, T. Vegge and D. J. Siegel, Chem. of Mater., 2019, 31, 9156-9167.

4 P. Hartmann, C. L. Bender, M. Vračar, A. K. Dürr, A. Garsuch, J. Janek and P. Adelhelm, Nat. Mater., 2013, 12, 228-32.

5 J. Lu, Y. Jung Lee, X. Luo, K. Chun Lau, M. Asadi, H.-H. Wang, S. Brombosz, J. Wen, D. Zhai, Z. Chen, D. J. Miller, Y. Sub Jeong, J.-B. Park, Z. Zak Fang, B. Kumar, A. SalehiKhojin, Y.-K. Sun, L. A. Curtiss and K. Amine, Nature, 2016, 529, 377-382.

6 M. Ziegler, M. Rosenfeld, W. Känzig and P. Fischer, Helv. Phys. Acta, 1976, 49, 57-90.

7 W. Hesse, M. Jansen and W. Schnick, Prog. Solid State Chem., 1989, 19, 47-110.

8 G. Kemeny and S. D. Mahanti, Phys. Rev. B, 1979, 20, 29612963.

9 S. D. Mahanti and G. Kemeny, Phys. Rev. B, 1979, 20, 21052117.

10 S. D. Mahanti and D. Sahu, Phys. Rev. Lett., 1982, 48, 936939.

11 F. Luty and J. Ortiz-Lopez, Phys. Rev. Lett., 1983, 50, 12891292.

12 S. D. Mahanti, G. Zhang and G. Kemeny, Phys. Rev. B, 1987, 35, 8551-8561.

13 R. M. Lynden-Bell and K. H. Michel, Rev. Mod. Phys., 1994, 66, 721-762.

14 P. Singh and N. K. Gaur, Phys. Lett. A, 2007, 371, 349-353.

15 M. Hayyan, M. A. Hashim and I. M. Alnashef, Chem. Rev., 2016, 116, 3029-3085.

16 H. Zeller and W. Känzig, Helv. Phys. Acta, 1967, 40, 845.

17 W. Känzig and M. H. Cohen, Phys. Rev. Lett., 1959, 3, 509510.

18 A. Devonshire, Proc. R. Soc. London. Ser. A - Math. Phys. Sci., 1936, 153, 601-621.

19 H. U. Beyeler, Phys. Status Solidi, 1972, 52, 419-425.

20 S. Estreicher and T. L. Estle, Phys. Rev. B, 1984, 30, 7-18.

21 S. D. Mahanti, P. Murray and G. Kemeny, Phys. Rev. B, 1985, 32, 3263-3271.

22 C. S. Ewig and J. Tellinghuisen, J. Chem. Phys., 1991, 95, 1097-1106.

23 C. Di Valentin and G. Pacchioni, Model. Simul. Mater. Sci. Eng., 2009, 17, 084005.

24 W. Känzig, Phys. Rev. Lett., 1961, 7, 304-306.

25 W. Känzig, J. Phys. Chem. Solids, 1962, 23, 479-499.

26 G. Pfister and W. Känzig, Phys. der Kondens. Mater., 1969, 10, 231-264.

27 I. Bersuker, The Jahn-Teller Effect, Cambridge University Press, 
Cambridge, 2006.

28 K. H. Michel and J. M. Rowe, Phys. Rev. B, 1980, 22, 14171428.

29 H. U. Beyeler, R. Baumann and W. Känzig, Phys. Konden. Mater., 1970, 11, 286-316.

30 K. K. Rebane and L. A. Rebane, Pure Appl. Chem., 1974, 37, 161-181.

31 I. B. Bersuker, Chem. Rev., 2001, 101, 1067-1114.

32 A. Larsen, J. Mortensen, J. Blomqvist, I. Castelli, R. Christensen, M. Dulak, J. Friis, M. Groves, B. Hammer, C. Hargus, E. Hermes, P. Jennings, P. Jensen, J. Kermode, J. Kitchin, E. Kolsbjerg, J. Kubal, K. Kaasbjerg, S. Lysgaard, J. Maronsson, T. Maxson, T. Olsen, L. Pastewka, A. Peterson, C. Rostgaard, J. Schiøtz, O. Schütt, M. Strange, K. Thygesen, T. Vegge, L. Vilhelmsen, M. Walter, Z. Zeng and K. W. Jacobsen, J. Phys. Condens. Matter, 2017, 29, 273002.

33 G. Kresse and J. Furthmüller, Phys. Rev. B, 1996, 54, 1116911186.

34 P. E. Blöchl, Phys. Rev. B, 1994, 50, 17953-17979.

35 J. P. Perdew, K. Burke and M. Ernzerhof, Phys. Rev. Lett., 1996, 77, 3865-3868.

36 J. Sun, A. Ruzsinszky and J. Perdew, Phys. Rev. Lett., 2015, 115, 036402.

37 H. J. Monkhorst and J. D. Pack, Phys. Rev. B, 1976, 13, 51885192.
38 J. M. García-Lastra, J. A. Aramburu, M. T. Barriuso and M. Moreno, Phys. Rev. Lett., 2004, 93, 226402.

39 C. Kittel, Introduction to Solid State Physics, Wiley, 2004.

40 G. Pfister and M. Bösch, J. Phys. Chem. Solids, 1970, 31, 2699-2707.

41 J. M. García-Lastra, M. T. Barriuso, J. A. Aramburu and M. Moreno, Chem. Phys., 2005, 317, 103-110.

42 J. Grammatikakis, M. Lazaridou and K. Eftaxias, J. Phys. Chem. Solids, 1985, 46, 1243-1246.

43 A. Bohm, A. Mostafazadeh, H. Koizumi, Q. Niu and J. Zwanziger, in Crossing of Potential Energy Surfaces and the Molecular Aharonov-Bohm Effect, Springer Berlin Heidelberg, Berlin, Heidelberg, 2003, pp. 195-224.

44 A. V. Krukau, O. A. Vydrov, A. F. Izmaylov and G. E. Scuseria, J. Chem. Phys., 2006, 125, 224106.

45 F. Bridges, C R C Crit. Rev. Solid State Sci., 1975, 5, 1-88.

46 L. Pauling, Phys. Rev., 1930, 36, 430-443.

47 T. Kiljunen, B. Schmidt and N. Schwentner, Phys. Rev. A, 2005, $72,053415$.

48 T. Kiljunen, B. Schmidt and N. Schwentner, J. Chem. Phys., 2006, 124, 164502.

49 P. García-Fernández, L. García-Canales, J. M. García-Lastra, J. Junquera, M. Moreno and J. A. Aramburu, J. Chem. Phys., 2008, 129, 124313. 